\title{
Dementia is Associated with an Increased Risk of Hip Fractures: A Nationwide Analysis in Korea
}

\author{
Jae-Hyuen Jeon ${ }^{\mathrm{a}}$ \\ Jeong Hoon Park ${ }^{\mathrm{b}}$ \\ Chungkun $\mathrm{Oh}^{\mathrm{b}}$ \\ Jae Kyung Chung ${ }^{b}$ \\ Jeong Yun Song ${ }^{\mathrm{b}}$ \\ Seongheon Kim ${ }^{b}$ \\ Seung-Hwan Lee ${ }^{b}$ \\ Jae-Won Jang ${ }^{\mathrm{b}}$ \\ Young-Ju Kim ${ }^{\mathrm{a}}$ \\ ${ }^{a}$ Department of Statistics, \\ Kangwon National University, \\ Chuncheon, Korea \\ ${ }^{b}$ Department of Neurology, \\ Kangwon National University \\ School of Medicine, \\ Kangwon National University Hospital, \\ Chuncheon, Korea
}

Background and Purpose Dementia has been reported to be associated with an increased risk of hip fracture, but few case-control studies have been performed to actually confirm this. This study investigated the association between dementia and hip fracture by comparing the risk of hip fracture between subjects with and without dementia.

Methods We examined a population-based matched cohort from the National Health Insurance Service-Senior Cohort data set that covers approximately half a million recipients of medical insurance in South Korea. Subjects with new clinically verified dementia during 2003-2007 were included, and five subjects without dementia were matched for age, sex, and index year to each subject with dementia as the controls. The risk of hip fracture for dementia was evaluated up to 2015 using Cox regression analysis.

Results During the 12-year follow-up period, 284 subjects with dementia (10.66\%) and 603 subjects without dementia (4.53\%) experienced hip fractures. Dementia was independently associated with a higher risk of hip fracture [hazard ratio $(\mathrm{HR})=2.840,95 \% \mathrm{CI}=2.449-3.293$ ] and the adjusted HR for hip fracture in the subjects with dementia was highest within 2 years after the initial diagnosis $(\mathrm{HR}=2.862,95 \% \mathrm{CI}=2.053-3.990)$.

Conclusions This study found that dementia could be an independent risk factor for hip fracture even at the early stage. This necessitates consideration of the future risk of falls and balance deficits in terms of physical activity after a diagnosis of dementia.

Key Words dementia, fracture, hip.

\section{INTRODUCTION}

Received October 18, 2018

Revised December 10, 2018

Accepted December 13, 2018

\section{Correspondence}

Jae-Won Jang, MD

Department of Neurology,

Kangwon National University

School of Medicine,

Kangwon National University Hospital,

156 Baengnyeong-ro,

Chuncheon 24289, Korea

Tel +82-33-258-9174

Fax +82-33-258-2103

E-mail light26@kangwon.ac.kr

Young-Ju Kim, PhD

Department of Statistics,

Kangwon National University,

1 Gangwondaehak-gil,

Chuncheon 24341, Korea

Tel +82-33-258-8431

Fax +82-33-259-5663

E-mail ykim7stat@kangwon.ac.kr
Dementia and fractures are two common problems that are frequently encountered in elderly people. ${ }^{1}$ Both are associated with increased mortality and morbidity as well as medical costs for the patients and caregivers. The number of individuals with dementia is estimated to double every 20 years and will reach approximately 40 million worldwide by 2020 due to the aging of the world population. ${ }^{2}$ Most hip fractures occur over the age of 65 years, and their total number is expected to increase to 6.3 million by $2050 .{ }^{3,4}$ Dementia is reportedly associated with increased falls that often lead to fractures. ${ }^{1,5}$ Since patients with dementia have a worse prognosis after a hip fracture ${ }^{6}$ with a higher mortality rate, ${ }^{7}$ it is important to understand the association between dementia and hip fractures in order to develop effective hip fracture preventive strategies.

Patients with dementia have a high risk of falling, and recent studies have revealed an association between cognitive decline and the risk of hip fracture. ${ }^{8-11}$ The relationship between dementia and hip fracture has been partly explained by their shared risk factors such as old age, ${ }^{12,13}$ female sex, ${ }^{14,15}$ and decreased activity. ${ }^{1}$ Furthermore, other mediating factors

(a) This is an Open Access article distributed under the terms of the Creative Commons Attribution Non-Commercial License (https://creativecommons.org/licenses/by-nc/4.0) which permits unrestricted non-commercial use, distribution, and reproduction in any medium, provided the original work is properly cited. 
such as falls, ${ }^{16,17}$ osteoporosis, ${ }^{14}$ or cachexia ${ }^{18}$ might increase the risk of hip fracture. In addition to these risks, patients with dementia are more likely to be taking medications such as antidepressants, antipsychotics, and anxiolytics, which might also increase the risk of falls. ${ }^{1}$

However, most previous studies have been performed with restricted cohorts, and large representative data sets involving long-term follow-up are rare. ${ }^{8,9}$ The purpose of the current study was to elucidate whether dementia is an independent risk factor for hip fracture and whether the fracture risk would differ over a long-term observational period using nationwide data in Korea.

\section{METHODS}

\section{Data acquisition}

The data set was provided by the Korean National Health Insurance Service (NHIS), which was founded in 2000 as a single-insurer system. The NHIS has integrated more than 366 of the medical insurance organizations in the Republic of Korea, and stored and converted medical records of individuals into a national database called the National Health Information Database. That database contains personal information, demographics, and medical treatment data for Korean citizens who were categorized as insured employees, insured selfemployed individuals, or medical aid beneficiaries.

The NHIS-Senior Cohort (NHIS-SC) is a research database for the elderly constructed in a cohort format for use when investigating the risk factors and prognoses of geriatric diseases. The NHIS-SC was constructed using a simple randomization method to extract data on 557,195 persons, representing $10 \%$ of the 5.5 million population aged 60 years or older, who had maintained their health-insurance and medical-care statuses at the end of December 2002.

The members of the NHIS-SC comprise a 14-year cohort (2002-2015) and are tracked in terms of socioeconomic variables (residence area, year and month of death, cause of death, and income level) and medical treatments (health examinations, medical-care history, and medical-care institutions). These data enable long-term observations and can be used to investigate causal relationships.

Diseases are registered in the NHIS-SC using the Korean Classification of Disease, sixth edition, which is modified from the International Classification of Disease, 10th revision (ICD10) for use by the NHIS and medical-care institutions in South Korea.

This study was approved by the Institutional Review Board of Kangwon National University Hospital (approval no. KNUH-2018-05-004-001).

\section{Study population}

This study involved a population-based matched cohort constructed using the NHIS-SC. Dementia was defined based on the ICD-10 classification codes (F00, F01, F02, F03, and G30) used with an antidementia drug (donepezil, galantamine, rivastigmine, or memantine) for the first or second diagnosis for medical expense claims up to December 31, 2007 through an outpatient clinic more than three times, or for more than one hospital admission. The index date for these subjects was defined as the date when dementia was first diagnosed after 2003. Hip fracture was defined as code S72.0 or S72.1 for the first or second diagnosis. We excluded subscribers with dementia registered in $2002(n=942)$ in order to identify newly diagnosed subjects with dementia, and subscribers with hip fractures before the diagnosis of dementia $(n=2,982)$ were also excluded in order to consider temporal relationships. Moreover, individuals who had stroke-related diagnoses (I60, I61, I62, I63, or I64; $n=24,472$ ) were also excluded before the index date to eliminate predisposing factors of vascular dementia.

We also used the body mass index (BMI) to analyze associations of the amount of tissue mass and dementia with hip fracture, because BMI influences the occurrence of hip fracture in the general population. ${ }^{19}$ When multiple BMI measurements were available for a single subscriber, the closest one to the time of registration was used. Those for whom BMI information was not available were excluded from the analysis $(n=165,064)$.

This study finally included 2,664 patients with dementia. For each subject in the case group, five subjects without dementia were randomly selected and matched for age, sex, and index date as controls (Fig. 1). ${ }^{20,21}$ Subjects in both groups were followed from the index date up to the occurrence of hip fracture, death, or December 31, 2015, whichever occurred first.

In addition to diagnoses, we extracted other variables including age, sex, BMI, residence area, and Charlson Comorbidity Index (CCI). Participants were subdivided based on the BMI into underweight $\left(<18.5 \mathrm{~kg} / \mathrm{m}^{2}\right)$, normal weight $(18.5-$ $\left.23.0 \mathrm{~kg} / \mathrm{m}^{2}\right)$, and overweight/obese $\left(>23.0 \mathrm{~kg} / \mathrm{m}^{2}\right)$ based on the generally accepted BMI criteria for Asians. ${ }^{22}$ In addition, based on the CCI, the severity of comorbidities was classified into four grades ( 0 for none, 1 or 2 for mild, 3 or 4 for moderate, and 5 for severe). The residence areas were classified into two types according to population size: metropolitan and nonmetropolitan.

\section{Statistical analysis}

The prevalence rate of dementia per 100,000 individuals in 2003 and the incidence rates of dementia per 100,000 per- 


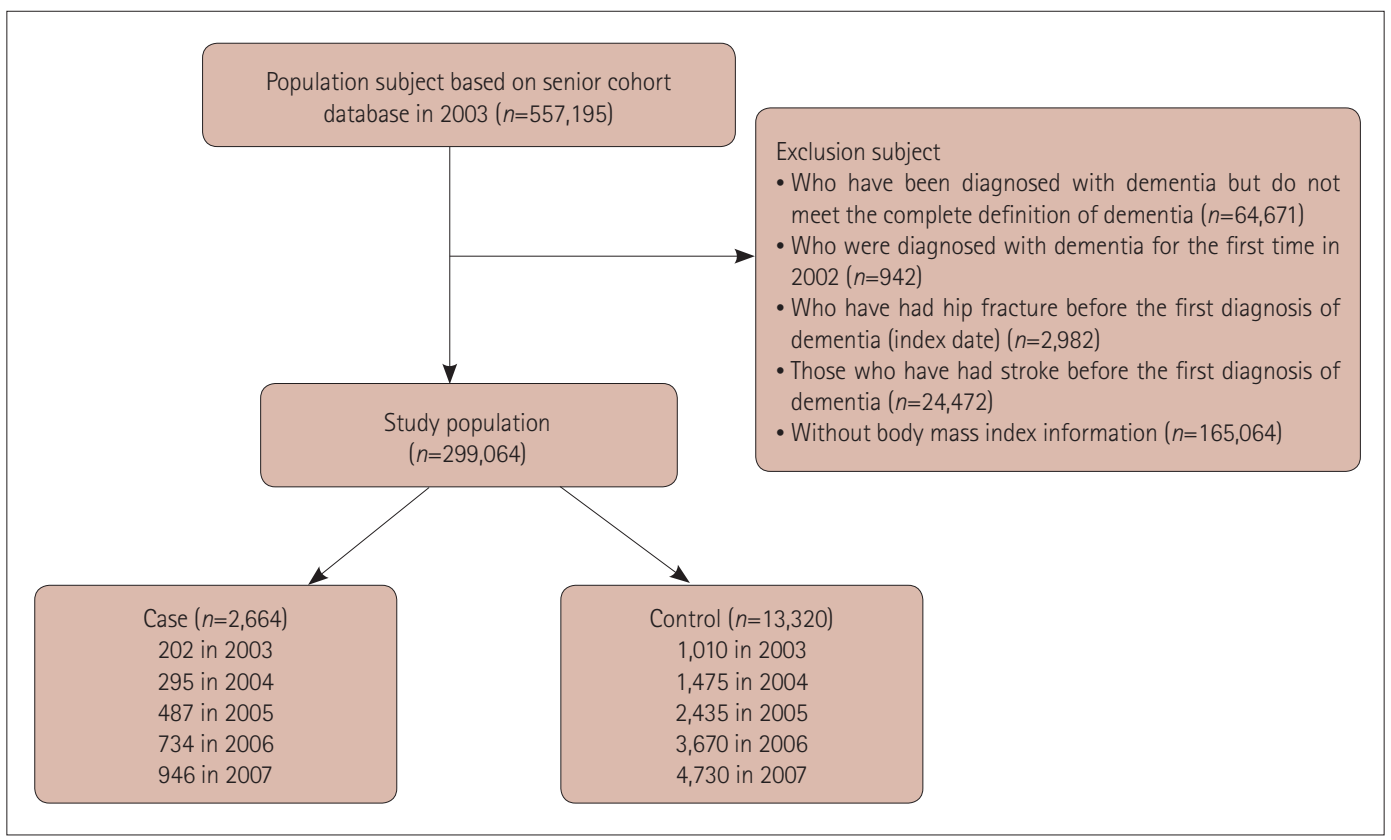

Fig. 1. Selection of cases and controls.

son-years during 2003 and 2015 were calculated. We used Pearson's chi-square test to compare differences in the rates of hip fractures between groups according to age, sex, BMI, residence area, and CCI. The Cox proportional-hazards model was used to calculate the adjusted hazard ratio (HR) and $95 \% \mathrm{CI}$ to determine whether dementia was an independent risk factor for hip fracture after adjusting for sex, age, BMI, residence area, and CCI. Survival time was defined as the duration from the index date up to a diagnosis of hip fracture or up to the censoring due to death or the end of the study on December 31, 2015, whichever occurred first. Those who were dead or were never diagnosed with a hip fracture by the end of the study were treated as censored. Kaplan-Meier survival curves were estimated, and the log-rank test was used to compare differences in the curves between two groups. The duration of dementia from the index date was arbitrarily divided into $<2,2-10$, and $>10$ years in order to compare differences in the relative risk of hip fracture between subjects with dementia and the controls. The statistical analyses were performed using the SAS for Windows statistical package (version 7.1, SAS Institute, Cary, NC, USA).

\section{RESULTS}

The BMI and the proportion of the population living in metropolitan areas were both lower in the dementia group than in the control group (Table 1). During the 12-year follow-up period, 284 subjects with dementia (10.66\%) and 603 subjects without dementia (4.53\%) experienced hip fractures (Table 2). Cox regression analysis showed that the risk of hip
Table 1. Distribution of the characteristics of the subjects in the retrospective matched cohort

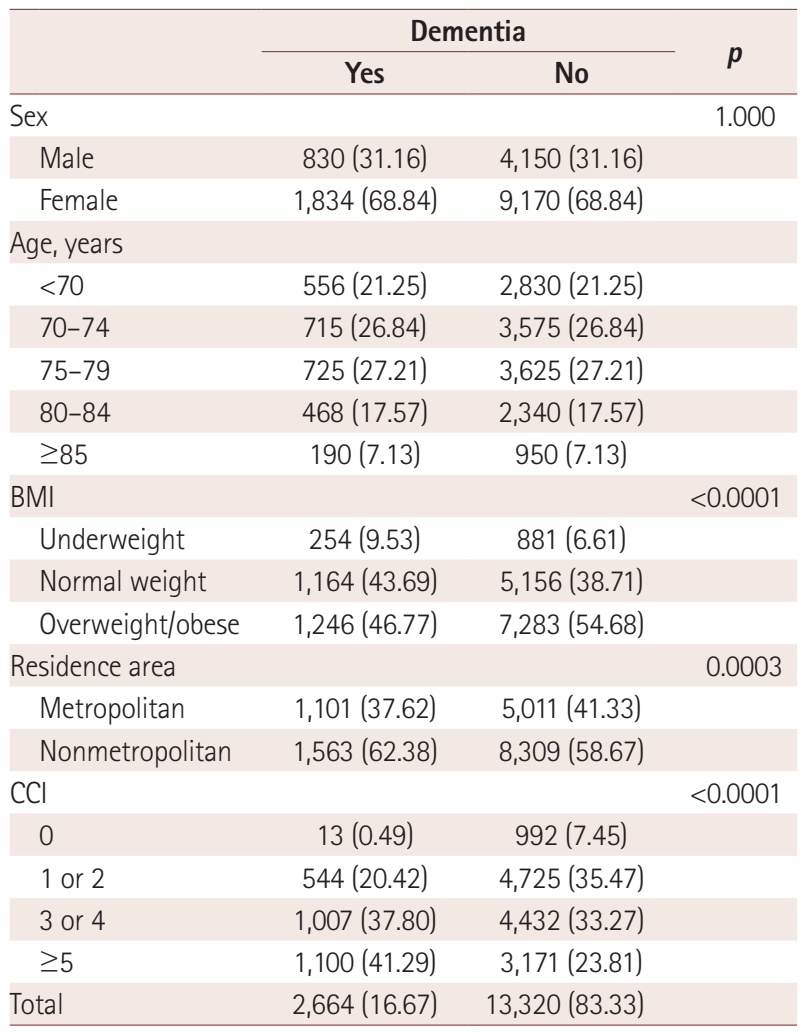

Data are $n(\%)$ values. $p$ value derived from Pearson's chi-square test. BMI: body mass index, CCl: Charlson Comorbidity Index.

fracture in the dementia group was 2.840-fold higher (adjusted HR, 95\% CI=2.449-3.293) than that in the control group. The risk of hip fracture in females was 1.715-fold high- 
Table 2. Incidence rate of hip fracture and adjusted HRs from the Cox proportional-hazards model

\begin{tabular}{|c|c|c|c|c|}
\hline & \multicolumn{2}{|c|}{ Hip fracture } & \multirow{2}{*}{$p$} & \multirow{2}{*}{ Adjusted HR $(95 \% \mathrm{Cl})$} \\
\hline & $n(\%)$ & IR & & \\
\hline Sex & & & $<0.0001$ & \\
\hline Male & $160(3.21)$ & 415.03 & & Reference \\
\hline Female & $727(6.61)$ & 773.48 & & $1.715(1.443-2.037)$ \\
\hline Age (years) & & & $<0.0001$ & \\
\hline$<70$ & $76(2.24)$ & 229.92 & & Reference \\
\hline $70-74$ & $181(4.22)$ & 469.19 & & 1.996 (1.526-2.611) \\
\hline $75-79$ & $273(6.28)$ & 774.31 & & $3.310(2.563-4.275)$ \\
\hline $80-84$ & $233(8.30)$ & 1190.45 & & 5.264 (4.049-6.844) \\
\hline$\geq 85$ & 124 (10.88) & 2039.42 & & $9.536(7.117-12.778)$ \\
\hline BMI & & & $<0.0001$ & \\
\hline Underweight & 102 (8.99) & 1308.08 & & $1.751(1.398-2.192)$ \\
\hline Normal weight & $398(6.30)$ & 790.29 & & $1.346(1.168-1.552)$ \\
\hline Overweight/obese & $387(4.54)$ & 520.28 & & Reference \\
\hline Residence area & & & 0.5901 & \\
\hline Metropolitan & $331(5.42)$ & 649.47 & & Reference \\
\hline Nonmetropolitan & $556(5.63)$ & 681.57 & & 1.038 (0.906-1.190) \\
\hline $\mathrm{CCl}$ & & & $<0.0001$ & \\
\hline 0 & $36(3.58)$ & 434.92 & & Reference \\
\hline 1 or 2 & $252(4.78)$ & 565.47 & & $1.223(0.861-1.736)$ \\
\hline 3 or 4 & $311(5.72)$ & 678.76 & & $1.423(1.004-2.017)$ \\
\hline$\geq 5$ & $288(6.74)$ & 850.03 & & $1.824(1.280-2.601)$ \\
\hline Dementia & & & $<0.0001$ & \\
\hline No & $603(4.53)$ & 526.69 & & Reference \\
\hline Yes & $284(10.66)$ & 1573.04 & & 2.840 (2.449-3.293) \\
\hline
\end{tabular}

$\mathrm{HR}$ was adjusted for sex, age, $\mathrm{BMI}$, residence area, and $\mathrm{CCl}, p$ value derived from Pearson's chi-square test.

BMI: body mass index, CCl: Charlson Comorbidity Index, Cl: confidence interval, HR: hazard ratio, IR: incidence rate per 100,000 person-years.

er (adjusted HR, 95\% CI=1.443-2.037) than that in males. The risk of hip fracture increased as age and CCI increased and BMI decreased, but did not differ between the two residence areas (Table 2). The Kaplan-Meier survival curves for the risk of hip fracture differed significantly between the two groups, and the risk of hip fracture was significantly higher in the dementia group than the control group (log-rank $p<$ 0.0001) (Fig. 2).

The adjusted HRs for hip fracture in patients with dementia $<2,2-10$, and $>10$ years after the diagnosis were $2.862(95 \%$ $\mathrm{CI}=2.053-3.990), 2.795$ (95\% CI=2.358-3.314), and 2.538 (95\% CI=1.276-5.047), respectively. The adjusted HRs showed a decreasing trend with increasing duration after the first diagnosis of dementia, and it was significant at all periods after adjusting for sex, age, BMI, CCI, and residence area (Table 3).

\section{DISCUSSION}

This study found a significant association between dementia and increased hip fracture after adjusting for confound-

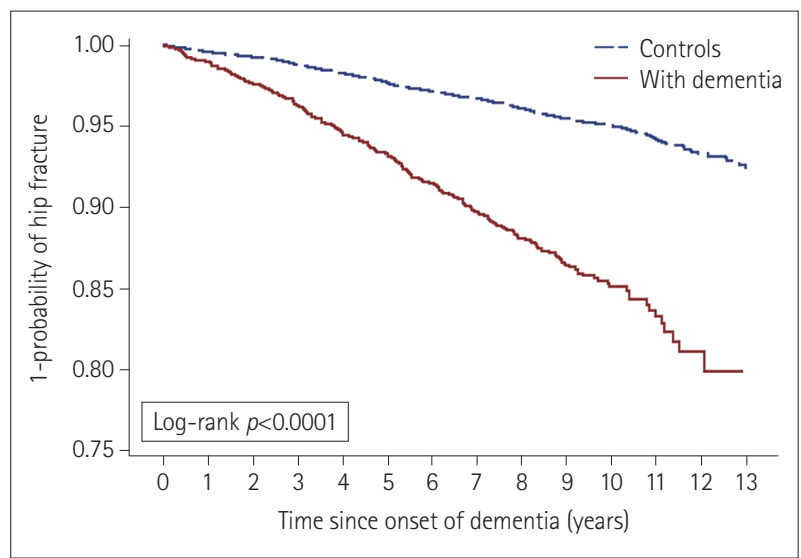

Fig. 2. Kaplan-Meier curves for hip fracture in the dementia and control groups.

ing factors using the NHIS-SC database, which contains information on approximately half a million elderly people. The risk of hip fracture was highest within 2 years after the diagnosis of dementia, and remained significantly higher compared to the control group. In general, female sex, older age, more comorbidities, and lower BMI were also found to be risk 
Table 3. HR values for hip fracture according to disease duration after the diagnosis

\begin{tabular}{|c|c|c|c|c|c|}
\hline & Dementia, $n(\%)$ & Control, $n(\%)$ & $\begin{array}{l}\text { Crude HR, } \\
(95 \% \mathrm{Cl})\end{array}$ & $\begin{array}{l}\text { Adjusted HR1, } \\
(95 \% \mathrm{Cl})\end{array}$ & $\begin{array}{l}\text { Adjusted HR2, } \\
(95 \% \mathrm{Cl})\end{array}$ \\
\hline \multicolumn{6}{|l|}{ Duration after diagnosis } \\
\hline Early (<2 years) & $60(2.25)$ & $98(0.74)$ & 3.155 (2.288-4.350) & $2.892(2.075-4.029)$ & $2.862(2.053-3.990)$ \\
\hline Middle (2-10 years) & 213 (8.96) & 452 (3.59) & $3.044(2.586-3.584)$ & $2.784(2.349-3.300)$ & $2.795(2.358-3.314)$ \\
\hline Late (>10 years) & $11(1.01)$ & $53(0.58)$ & 2.655 (1.379-5.111) & $2.492(1.256-4.945)$ & $2.538(1.276-5.047)$ \\
\hline Overall & $284(10.66)$ & $603(4.53)$ & 3.107 (2.695-3.581) & $2.853(2.461-3.307)$ & $2.859(2.466-3.315)$ \\
\hline
\end{tabular}

Adjusted HR1; HR adjusted for sex, age, BMI group, and CCl. Adjusted HR2; HR adjusted for sex, age, BMI group, CCl, and residence area. BMI: body mass index, CCl: Charlson Comorbidity Index, Cl: confidence interval, HR: hazard ratio.

factors for hip fracture in the elderly subjects.

We found that BMI, residence area, and CCI differed significantly between subjects with and without dementia at baseline (Table 2). A lower BMI is known to be associated with low bone mineral density ${ }^{19}$ and is more common in subjects with dementia than in controls. ${ }^{23}$ A significantly lower nutritional screening score was found in nonmalnourished subjects with very mild dementia, indicating that subtle differences in nutritional status are present even at the early stage of dementia. ${ }^{24}$ This nutritional imbalance could result in a risk of malnutrition in patients with lower BMI and longer dementia duration. ${ }^{25}$ Considering that the $\mathrm{CCI}$ comprises risk factors for dementia such as cardiovascular risk factors, it is consistent that the subjects with dementia had more comorbidities with a higher CCI-and hence requiring adjustment-than did the control subjects. ${ }^{26,27}$ Since the residence area was divided into metropolitan and nonmetropolitan areas, it did not reflect differences in the activity of subjects according to the detailed population distribution. However, since the characteristics of elderly people in metropolitan and nonmetropolitan areas might differ, it is meaningful that there were regional differences at baseline in the distribution of the patients with dementia.

Dementia was identified as an independent risk factor for hip fracture based on a long-term follow-up (Table 3). This is in line with the findings of previous studies suggesting that subjects with dementia have an increased risk of hip fracture. ${ }^{1,8,11,14,28,29}$ Patients with dementia are more likely to have osteoporosis due to lower bone marrow density and vitamin $\mathrm{D}$ deficiency, ${ }^{30,31}$ which would partly explain the higher risk of hip fracture found in the present study. Additionally, the association between hip fracture and dementia might be due to an increased rate of falls. Elderly patients with dementia have balance and gait deficits as well as autonomic dysfunction, which reportedly increases the risk of falls approximately twofold. ${ }^{29,32}$

The risk of hip fracture was higher in the subjects with dementia than the control subjects on longitudinal observation (Fig. 2), with the adjusted HR peaking within 2 years af- ter the diagnosis of dementia (Table 3). An index of disease severity such as the Clinical Dementia Rating was not included, and so this finding should be interpreted with caution. However, it is particularly interesting because the severity of dementia usually aggravates after a diagnosis, and a longer dementia duration supposedly increases the risk of hip fracture, being higher in subjects with moderate dementia than in those with mild impairment. ${ }^{33}$ In contrast to this expectation, our results indicate that a shorter dementia duration appears to be associated with a greater or similar risk of hip fracture. The gradual decrease in outside activity of patients with advanced dementia might be paradoxically related to this finding. ${ }^{34-36}$ Because of their loss of independence as well as depressive symptoms, ${ }^{37}$ patients with advanced dementia exhibit decreased outdoor activity, which might reduce their risk of fall-related hip fractures compared to the early stage of dementia. Moreover, there is increasing evidence that balance and gait deficits are present even at the early stage of dementia, ${ }^{32,38}$ and that the longitudinal interactions between physical and cognitive performance are likely to operate bidirectionally. ${ }^{39}$ The concomitant decline of cognition and physical function in patients with dementia that might result in fall-related hip fractures necessitates the early identification of mobility deficits.

There are several limitations of this study to consider. First, dementia and hip fracture were diagnosed based on ICD-10 codes, and thus selection bias may exist despite the adoption of the operational definition. We did not differentiate the subtypes of dementia because they are not clearly divided in claims data. Second, the NHIS-SC database does not include certain items of important information such as cognitive status as represented by the index score, APOE4 carrier status, or level of education, because the included data are based on claims and health screening data only. Therefore, the statistical analysis was performed without adjusting for these variables. Third, although osteoporosis is known to be a very important risk factor for fracture, ${ }^{40}$ the present study did not include data regarding the diagnosis and treatment of osteoporosis, and so the association between dementia and osteo- 
porosis was not analyzed.

One important strength of this study lies in its use of a long-term nationwide database system with a large population of patients with dementia compared to age- and sexmatched subjects without dementia, which will have minimized selection bias and maximized the statistical power. Most of the findings of our study match those of communitybased and meta-analytic studies of the association between dementia and hip fracture. ${ }^{8,29,41}$

In conclusion, the subjects with dementia had a significantly increased risk of hip fracture after adjusting for confounding factors. This study also found that the risk of hip fracture was highest within 2 years after the diagnosis of dementia. It is therefore important to educate patients regarding the risk of hip fracture starting at the early stage of the disease, as well as to implement preventive measures. Future studies could focus on the development of practical preventive measures and patient education.

\section{Conflicts of Interest}

The authors have no potential conflicts of interest to disclose.

\section{Acknowledgements}

This research was supported by the Original Technology Research Program for Brain Science through the National Research Foundation of Korea (NRF) funded by the Korean government (MSIP) (No. 2014M3C7A1064 752) and by the Korea Health Technology R\&D Project through the Korea Health Industry Development Institute (KHIDI), funded by the Ministry of Health \& Welfare, Republic of Korea (HI18C0479).

This study used National Health Insurance Service-Senior Cohort data (NHIS-2018-2-181), made by National Health Insurance Service.

\section{REFERENCES}

1. Friedman SM, Menzies IB, Bukata SV, Mendelson DA, Kates SL. Dementia and hip fractures: development of a pathogenic framework for understanding and studying risk. Geriatr Orthop Surg Rehabil 2010; 1:52-62.

2. Ferri CP, Prince M, Brayne C, Brodaty H, Fratiglioni L, Ganguli M, et al. Global prevalence of dementia: a Delphi consensus study. Lancet 2005;366:2112-2117.

3. Braithwaite RS, Col NF, Wong JB. Estimating hip fracture morbidity, mortality and costs. J Am Geriatr Soc 2003;51:364-370.

4. Marks R. Hip fracture epidemiological trends, outcomes, and risk factors, 1970-2009. Int J Gen Med 2010;3:1-17.

5. Amboni M, Barone P, Hausdorff JM. Cognitive contributions to gait and falls: evidence and implications. Mov Disord 2013;28:1520-1533.

6. Holmes J, House A. Psychiatric illness predicts poor outcome after surgery for hip fracture: a prospective cohort study. Psychol Med 2000; 30:921-929.

7. Baker NL, Cook MN, Arrighi HM, Bullock R. Hip fracture risk and subsequent mortality among Alzheimer's disease patients in the United Kingdom, 1988-2007. Age Ageing 2011;40:49-54.

8. Tolppanen AM, Lavikainen P, Soininen H, Hartikainen S. Incident hip fractures among community dwelling persons with Alzheimer's disease in a Finnish nationwide register-based cohort. PLoS One 2013;8: e59124.

9. Tolppanen AM, Taipale H, Tanskanen A, Tiihonen J, Hartikainen S. Comparison of predictors of hip fracture and mortality after hip frac- ture in community-dwellers with and without Alzheimer's disease exposure-matched cohort study. BMC Geriatr 2016;16:204.

10. Scandol JP, Toson B, Close JC. Fall-related hip fracture hospitalisations and the prevalence of dementia within older people in New South Wales, Australia: an analysis of linked data. Injury 2013;44:776-783.

11. Lai SW, Chen YL, Lin CL, Liao KF. Alzheimer's disease correlates with greater risk of hip fracture in older people: a cohort in Taiwan. J Am Geriatr Soc 2013;61:1231-1232.

12. Hebert LE, Scherr PA, Beckett LA, Albert MS, Pilgrim DM, Chown MJ, et al. Age-specific incidence of Alzheimer's disease in a community population. JAMA 1995;273:1354-1359.

13. Farmer ME, Harris T, Madans JH, Wallace RB, Cornoni-Huntley J, White LR. Anthropometric indicators and hip fracture. The NHANES I epidemiologic follow-up study. J Am Geriatr Soc 1989;37:9-16.

14. Weller I, Schatzker J. Hip fractures and Alzheimer's disease in elderly institutionalized Canadians. Ann Epidemiol 2004;14:319-324.

15. Johansson C, Skoog I. A population-based study on the association between dementia and hip fractures in 85-year olds. Aging (Milano) 1996; 8:189-196.

16. Allan LM, Ballard CG, Rowan EN, Kenny RA. Incidence and prediction of falls in dementia: a prospective study in older people. PLoS One 2009; 4:e5521.

17. Anstey KJ, Von Sanden C, Luszcz MA. An 8-year prospective study of the relationship between cognitive performance and falling in very old adults. J Am Geriatr Soc 2006;54:1169-1176.

18. Poehlman ET, Dvorak RV. Energy expenditure, energy intake, and weight loss in Alzheimer disease. Am J Clin Nutr 2000;71:650S-655S.

19. De Laet C, Kanis JA, Odén A, Johanson H, Johnell O, Delmas P, et al. Body mass index as a predictor of fracture risk: a meta-analysis. Osteoporos Int 2005;16:1330-1338.

20. Kripke DF, Langer RD, Kline LE. Hypnotics' association with mortality or cancer: a matched cohort study. BMJ Open 2012;2:e000850.

21. Rim TH, Kim DW, Han JS, Chung EJ. Retinal vein occlusion and the risk of stroke development: a 9-year nationwide population-based study. Ophthalmology 2015;122:1187-1194.

22. Anuurad E, Shiwaku K, Nogi A, Kitajima K, Enkhmaa B, Shimono K, et al. The new BMI criteria for asians by the regional office for the western pacific region of WHO are suitable for screening of overweight to prevent metabolic syndrome in elder Japanese workers. J Occup Health 2003;45:335-343.

23. Faxén-Irving G, Fereshtehnejad SM, Falahati F, Cedergren L, Göranzon $\mathrm{H}$, Wallman $\mathrm{K}$, et al. Body mass index in different dementia disorders: results from the Swedish dementia quality registry (SveDem). Dement Geriatr Cogn Dis Extra 2014;4:65-75.

24. Olde Rikkert MG, Verhey FR, Sijben JW, Bouwman FH, Dautzenberg PL, Lansink M, et al. Differences in nutritional status between very mild Alzheimer's disease patients and healthy controls. J Alzheimers Dis 2014;41:261-271

25. Droogsma E, Van Asselt DZ, Schölzel-Dorenbos CJ, Van Steijn JH, Van Walderveen PE, Van der Hooft CS. Nutritional status of communitydwelling elderly with newly diagnosed Alzheimer's disease: prevalence of malnutrition and the relation of various factors to nutritional status. J Nutr Health Aging 2013;17:606-610.

26. Van der Flier WM, Scheltens P. Epidemiology and risk factors of dementia. J Neurol Neurosurg Psychiatry 2005;76 Suppl 5:v2-v7.

27. Gorelick PB. Risk factors for vascular dementia and Alzheimer disease. Stroke 2004;35(11 Suppl 1):2620-2622.

28. Nordström P, Franks PW, Gustafson Y, Nordström A. Cognitive function in young men and the later risk of fractures.J Bone Miner Res 2012; 27:2291-2297.

29. Wang HK, Hung CM, Lin SH, Tai YC, Lu K, Liliang PC, et al. Increased risk of hip fractures in patients with dementia: a nationwide population-based study. BMC Neurol 2014;14:175.

30. Zhao Y, Shen L, Ji HF. Alzheimer's disease and risk of hip fracture: a meta-analysis study. ScientificWorldJournal 2012;2012:872173. 
dementia on outdoor life: a 'shrinking world'? Dementia 2008;7:191204.

37. Paterniti S, Verdier-Taillefer MH, Dufouil C, Alpérovitch A. Depressive symptoms and cognitive decline in elderly people. Longitudinal study. Br J Psychiatry 2002;181:406-410.

38. Wilkins $\mathrm{CH}$, Roe CM, Morris JC, Galvin JE. Mild physical impairment predicts future diagnosis of dementia of the Alzheimer's type. $J$ Am Geriatr Soc 2013;61:1055-1059.

39. Tolea MI, Morris JC, Galvin JE. Longitudinal associations between physical and cognitive performance among community-dwelling older adults. PLoS One 2015;10:e0122878.

40. Gleason LJ, Menzies IB, Mendelson DA, Kates SL, Friedman SM. Diagnosis and treatment of osteoporosis in high-risk patients prior to hip fracture. Geriatr Orthop Surg Rehabil 2012;3:79-83.

41. Norring-Agerskov D, Laulund AS, Lauritzen JB, Duus BR, Van der Mark S, Mosfeldt M, et al. Metaanalysis of risk factors for mortality in patients with hip fracture. Dan Med J 2013;60:A4675. 